\begin{tabular}{|c|l|}
\hline Title & Mechanism of spatially resolved photochemical control of the resistivity of a molecular crystalline solid \\
\hline Author(s) & Naito, T.; Sugawara, H.; Inabe, T. \\
\hline Citation & $\begin{array}{l}\text { Nanotechnology, 42(18), 424008 } \\
\text { https://doi.org/L0.1088/0957-4484/48/42/424008 }\end{array}$ \\
\hline Issue Date & 2007-10-24 \\
\hline Doc URL & http://hdl.handle.net/2115/28257 \\
\hline Rights & Copyright $\odot$ 2007 IOP Publishing Ltd. \\
\hline Type & article (author version) \\
\hline File Information & NNT18-42.pdf \\
\hline
\end{tabular}

Instructions for use 


\title{
Mechanism of spatially resolved photochemical control of resistivity of a molecular crystalline solid
}

\author{
T Naito $^{1,2,3}$, H Sugawara ${ }^{2}$ and T Inabe ${ }^{2}$ \\ ${ }^{1}$ CRIS, Hokkaido Univ., Sapporo, Hokkaido 001-0021, Japan \\ ${ }^{2}$ Graduate School of Science, Hokkaido University, Sapporo, Hokkaido \\ 060-0810, Japan \\ E-mail: tnaito@mail.cris.hokudai.ac.jp
}

\begin{abstract}
By maintaining $\alpha-\mathrm{Ag}(\mathrm{DM})_{2}\left(\mathrm{DM}=\mathrm{C}_{10} \mathrm{H}_{8} \mathrm{~N}_{4}\right)$ around room temperature $(\mathrm{RT})\left(<<155^{\circ}\right.$

C) during UV-Vis irradiation, a series of photochemical products named $\beta$ was obtained. The solid $\beta$ was comprised of structurally different compounds. All of the solid $\beta$ phases share the original chemical formula of $\operatorname{Ag}(\mathrm{DM})_{2}$ and exhibit semiconducting behavior, yet differ from each other in their XRD patterns. They are divided into two phases: $\beta 1$ and $\beta 2$. Compared with the XRD pattern of $\alpha, \beta 1$ exhibited XRD peaks at the same $2 \theta$-angles but with different relative intensities, while $\beta 2$ exhibited totally different XRD patterns from those of $\alpha$ or $\beta 1$. The actual composition of a particular solid of “ $\beta$ ” is dependent upon irradiation time, and can be controlled continuously. Around RT, $\alpha$ exhibits metallic conductivity, while $\beta 1$ and $\beta 2$ are suggested to be semiconductors. As a result, one can continuously and finely control the electrical resistivity of $\mathrm{Ag}(\mathrm{DM})_{2}$ by $\mathrm{UV}$ irradiation to modify it into a mixture of $\alpha, \beta 1$ and/or $\beta 2$.
\end{abstract}

PACS: 72.20.-i; 72.80.-r; 72.80.Ng; 72.90.+y

\section{Introduction}

In the field of functional materials, keen attention has been paid to molecular materials [1-3]. Molecular materials possess a large number of unique properties, including a wide choice

${ }^{3}$ Author to whom any correspondence should be addressed. 
of processing methods under ambient conditions, the ability to self-aggregate, a well-established material design and a rich variation in materials. Two main trends in basic electronic research based on molecular materials take advantage of these unique properties. One of these trends includes the development of field effect transistors (FETs) and electroluminescence (EL) displays. The key differences from existing devices lie in the different goals and usages of the resultant electronic products. For example, some researchers fabricated large-area sensors and actuators, which have not been realized with present silicon-based electronics alone [4]. Also, many of them do not require highly elaborate fabrication techniques such as nanoscale integrated circuits, though they are still in the prototype stage. The other trend is illustrated in research on devices based on carbon nanotubes or single-molecule junctions, where a minimum unit of device function is condensed into a single or a few molecule(s) [5-12].

The research field of "molecular" or "organic" electronics maintains these two trends as their representative targets, i.e., organic FET/EL or single molecule devices. However, there is a different point of view in studying future non-silicon-based devices. Among molecular materials, crystalline charge transfer salts form a unique group in that many of them originally have sufficient conductivity with low-dimensional crystal/electronic structures. This means that they should have nano-structured and well-defined conduction pathways associated with their crystal structures. Their conducting properties are highly anisotropic in general, and thus they are described as "low-dimensional" conductors, meaning that electrical conduction does not occur in a three-dimensional way in the crystal, but only in particular directions. Due to quantum effects, such a high anisotropy or low-dimensionality leads to unconventional structural/electrical/magnetic properties that other materials with three-dimensional band structures never exhibit [1]. Such unique properties, however, cannot fully manifest themselves in bulk electrical properties when the materials are fabricated into thin films, because the original molecular arrangements and anisotropy of band structures are disturbed and averaged in an inhomogeneous and ill-defined way.

If it were possible to control the electronic structures of arbitrary parts of single crystals of molecular conductors, then the above-mentioned properties could be exploited by fabricating these parts into junction-structures and then devices. The performance of such devices is expected to surpass that of thin-film devices. As for inorganic devices, control of their conduction properties is well established by doping. There are many known methods for doping inorganic materials including ion implantation, laser doping and plasma doping. However, none of them are applicable to molecular materials because they are too fragile/unstable to survive such mechanically and thermally severe conditions. Chemical doping based on simple mixing or diffusion methods is also used in silicon technology, and could be applicable to molecular materials under ambient conditions [13]. The problem in these cases, however, is the difficulty in 
controlling the amount and distribution of dopants introduced in the host solids. Recently a possible solution to these kinds of doping problems is proposed; conductivity of a molecular charge transfer salt $\left[\mathrm{Ag}(\mathrm{DM})_{2} ; \mathrm{DM}=\mathrm{C}_{10} \mathrm{H}_{8} \mathrm{~N}_{4}\right]$ has been controlled using a photochemical solid state reaction [14].

A large number of molecular conductors have been reported to date and are well characterized in their crystalline states [1, 15-18]. Some of them contain photosensitive chemical species such as silver ions, which are not involved in the conduction pathways. These species could play a role resembling the "charge reservoir" of copper oxide high- $T_{c}$ superconductors [19]. Such molecular solids are generally stable under ambient conditions. However, they could be reactive under irradiation with ultraviolet and visible (UV-Vis) light, where electron transfer could occur between the photosensitive and the conducting molecular species. As a result, irradiation could irreversibly increase or decrease the amount of conducting electrons by adding or removing them from the conduction pathways. By analogy with "carrier doping" and to distinguish this phenomenon from "photodoping” [20], this is tentatively called "optical doping”. When only an arbitrary part of the sample was irradiated, the resistivity change occurred exclusively in the affected part, and the remaining sample retained its original metallic behavior. In combination with photolithography or light-focusing techniques, this may open up a new way to control electrical properties of a particular part of a molecular crystal irreversibly, which would lead to fabrication of devices with junction-structures.

The mechanism of this "optical doping" at first appeared to be irreversible photo-induced electron transfer between $\mathrm{Ag}^{+}$ions and the acceptor radical anion species which gradually decomposed the compound to domains of bulk silver. Actually, X-ray photoelectron spectroscopy (XPS) measurements detected bulk silver on surfaces of $\mathrm{Ag}(\mathrm{DM})_{2}$ after irradiation with UV-Vis light [14]. However, further study found that UV-Vis irradiation on $\operatorname{Ag}(\mathrm{DM})_{2}$ brought about many complicated reactions depending on the irradiation conditions [21], and above-mentioned observations turned out to originate from a mixture of the different photochemical products. Approximately with increasing the light in intensity and duration, there are four kinds of photochemical products derived from the pristine $\mathrm{Ag}(\mathrm{DM})_{2}$. For simplicity, the pristine crystalline material and all the photochemical products are serially called $\alpha, \beta, \gamma, \delta$, and $\varepsilon$, respectively below. They are clearly distinguished from each other in terms of chemical, physical, structural and spectroscopic properties. Except for $\beta$, all of them have their own well-defined homogeneous compositions. All the transformation is possible directly from $\alpha$ and are irreversible. Both $\beta$ and $\gamma$ retain the original chemical composition of $\operatorname{Ag}(\mathrm{DM})_{2}$, but $\gamma$ is an insulating, transparent, and amorphous solid. In $\gamma$ the molecular structure of DM is completely retained and every charge on the $\mathrm{Ag}^{+}$ions remains unchanged. The only microscopic structural difference between $\alpha$ and $\gamma$ lies in the coordination chemistry of $\mathrm{Ag}^{+}$ions; two of the four 
coordination bonds (Ag-DM) originally existing in the crystalline phase $\alpha$ are broken, which was indicated by chemical and spectroscopic analyses including EXAFS (Extended X-ray absorption fine structure) [21]. Thus $\gamma$ does not contain domains of isolated (liberated) neutral silver species; all the silver should be DM-coordinated monocationic species $\mathrm{Ag}^{+}$. The remaining two products, $\delta$ and $\varepsilon$, are products after net charge (electron) transfer from the DM to the Ag species followed by chemical decomposition. They are designated as $\operatorname{Ag}(\mathrm{DM})_{\mathrm{x}}(\mathrm{x} \approx 1.5)(\delta)$ and $\operatorname{Ag}(\varepsilon)$, respectively. More precisely, $\delta$ is better described as silver nanoparticles dispersed in neutral organic molecular (DM) amorphous matrix, and there is no component metal-complex molecule in the solid such as $\operatorname{Ag}(D M)_{x}(x \approx 1.5)$. All the four products appear to be metastable; once they are produced and irradiation is ceased, they are not transformed to further irradiated states on resuming irradiation or even with stronger light for a prolonged time. However, thermochemical measurements proved that heating in air could transform any of them finally into $\varepsilon$, i.e. elemental silver. Therefore the detected silver by XPS above [14] is now considered to have been produced mainly by heating effect involved with UV-Vis irradiation in air, and not necessarily relevant to photochemical resistivity change of $\alpha$. The main subject of this paper is $\beta$, which shares many of the chemical, structural and spectroscopic properties with those of $\alpha$ except for the conducting behavior; $\alpha$ is metallic while $\beta$ is semiconducting. Furthermore, XAFS (X-ray absorption fine structure) and XPS studies have recently revealed that $\beta$ should not contain elemental silver domains. In other words, control of resistivity by irradiation can be realized in $\beta$ without a cost of partial decomposition to bulk silver. Accordingly, one cannot always relate the following two facts: a) photo-induced electron transfer and resultant change in the electrical resistivity, and b) decomposition of the compound with loss of its crystalline structure. In this paper, we discuss these phenomena based on the chemical, physical, thermal, structural and spectroscopic properties of the photochemical products.

\section{Experimental Section}

The silver salt of 2,5-dimethyl- $N, N$ '-dicyanoquinonediimine, $\operatorname{Ag}(\mathrm{DM})_{2}$, was prepared according to the literature [22].

Infrared (IR) spectra were measured on $\mathrm{KBr}$ disks with a Perkin Elmer Spectrum One FT-IR spectrometer.

High resolution solid state ${ }^{13} \mathrm{C}-\mathrm{NMR}$ spectra were measured by means of cross polarization magic angle spinning (CPMAS) using a conventional pulsed NMR spectrometer JEOL ECX 400 at room temperature (RT). The sample contained ${ }^{13} \mathrm{C}$ nuclei at natural abundance. 
The NMR frequency was $395.9 \mathrm{MHz}$, corresponding to $9.3 \mathrm{~T}$. Sidebands were recognized by comparison of spectra from independent measurements with different sample spinning rates.

MALDI (matrix-assisted laser desorption ionization) mass spectra were measured using Applied Biosystems Voyager $\mathrm{DE}^{\mathrm{TM}}$-STR. The simplest spectra were obtained when no matrix was used in all the solid samples; most of the standard matrix compounds reacted with the original and/or fragment species to give complicated spectra with poor reproducibility.

Elemental analyses were carried out at the Center for Instrumental Analysis, Hokkaido University.

A $\mathrm{Hg} / \mathrm{Xe}$ lamp, described previously, was used [21, 23, 24]. In the following experiments, the power of light was fixed to be $300 \mathrm{mWcm}^{-2}$ at the sample and only the UV region of 254-450 nm was used. Well ground samples were irradiated while suspended and stirred in water. This method enabled both easy control of temperature and homogeneous irradiation. In electrical resistivity measurements during irradiation, which could not be conducted in water, the single crystals with four probes attached were exposed to UV light. Through water or air, irradiation provided the samples with an approximately similar strength of light. The possible effects of water, oxygen and other species on the samples were checked by XPS, Auger electron spectroscopy (AES), XAFS, IR, ${ }^{13} \mathrm{C}-\mathrm{NMR}$, scanning electron microscopy (SEM), differential scanning calorimetry (DSC) and elemental analysis. The results (not shown) indicated that deterioration, if any, should be below the detection limits.

The electrical resistivity measurements of single crystals of $\alpha$ and $\beta$ were carried out using a standard 4-probe method using gold wires ( $25 \mu \mathrm{m}$ in diameter) and gold paste (No.8560, Tokuriki Chemical Research Co., Ltd.) as electrical contacts. A home-made cryostat and other equipment were used to measure the resistivity at lower temperature. Details of the electrical measurements have been described in a previous paper [25]. The entire crystal surface (a black fine needle typically $\sim 1-2 \mathrm{~mm}$ long, $\sim 0.1 \mathrm{~mm}$ wide and $\sim 0.02 \mathrm{~mm}$ thick) was continuously illuminated with UV light at $20{ }^{\circ} \mathrm{C}$. Parallel measurements of the pristine samples were also conducted for determining the presence of any artifacts.

X-ray diffraction (XRD) patterns were measured with a RIGAKU X-ray Diffractometer RINT-Ultima+. $\mathrm{Cu}-\mathrm{K}_{\alpha}$ radiation ( $\lambda=1.540562 \AA$ ) was used with the Debye-Scherrer method. Filters were not used. The camera diameter was $285 \mathrm{~mm}$. The diffracted X-rays were detected with a scintillation counter. White radiation was used as the incident beam and the diffracted $\mathrm{X}$-ray was monochromated with a bent single crystal of graphite before detection. The finely ground powder samples were mounted on a glass plate on the sample stage. On the same samples used in the XRD study, some of the $\beta$ phase were also examined by XAFS on Ag in order to relate the local structures and chemical states with XRD patterns. For this purpose, these samples were mounted on the XRD sample stage sealed in Scotch tape. 


\section{Results and discussion}

The $\mathrm{Ag}(\mathrm{DM})_{2}$ compound discussed in this paper is a molecular charge-transfer complex between silver (Ag) and the $\pi$-electron acceptor DM (2,5-dimethyl- $N, N$ '-dicyanoquinonediimine) (figure 1a) [22,26]. Owing to its moderate electron accepting ability, DM is stable both as a neutral molecule and a radical anion species. When DM forms a salt with silver, charge (electron) transfer occurs between the DM molecules and silver atoms. As a result, every DM molecule formally exists as an anion radical species with $-1 / 2$ charges each, while the silver atoms become monocations, thus maintaining charge neutrality in the whole solid. In the crystal, the DM molecules stack to form a columnar structure due to $\pi-\pi$ intermolecular interactions (figure 1b) and unpaired electrons on the radical anion species delocalize along these columns, resulting in "one-dimensional" metallic conduction. All the DM molecules are bonded with the $\mathrm{Ag}^{+}$ions through the $=\mathrm{N}-\mathrm{CN}$ groups in $\mathrm{Ag}(\mathrm{DM})_{2}$. This structural feature connects the organic conduction columns and the $\mathrm{Ag}^{+}$ions. Generally, the typical energy of a covalent bond compares with that of UV-Vis light. Therefore, excitation by UV-Vis light on this material is considered to bring about electron transfer between the DM and silver ions. If this is irreversible as in a case accompanied by bond cleavage, it should involve a change in the number of unpaired electrons and thus the conductivity and magnetism, and even the crystal structure in some cases, will also change. UV-Vis irradiation can be expected to induce a complicated series of photochemical phenomena followed by structural local deformations and relaxations in $\mathrm{Ag}(\mathrm{DM})_{2}$. On the other hand, the infinite coordination network -DM-Ag-DMextended in the whole crystal should be robust and will resist structural change. Therefore, we examined how these conflicting structural situations affect the electrical and magnetic properties of $\mathrm{Ag}(\mathrm{DM})_{2}$ under UV irradiation.

Figure 2 shows the result of the continuous monitoring of resistivity of $\operatorname{Ag}(\mathrm{DM})_{2}$ during UV irradiation at RT. The irradiation commenced at $c a .700 \mathrm{~s}$ after the resistivity measurement started. Before the irradiation (< $700 \mathrm{~s}$ ) the resistivity was constant within experimental error. When the UV light was turned on, the resistivity suddenly increased. This could be attributed to heating effects involved with the irradiation. Afterward the resistivity gradually and steadily increased. In this experiment the UV light was turned off for 75 minutes to check whether the resistivity change was reversible. Without irradiation the resistivity instantly dropped and became constant, but the constant resistivity value was higher than the original value. When turning on the light again, the resistivity quickly returned to the value it had before turning off the light and resumed the preceding trend. From these results the origin of the resistivity increase could be separated 
factors: 
(a)<smiles>CC1=CC(=NC=N)C(C)=CC1=NC#N</smiles>

(b)

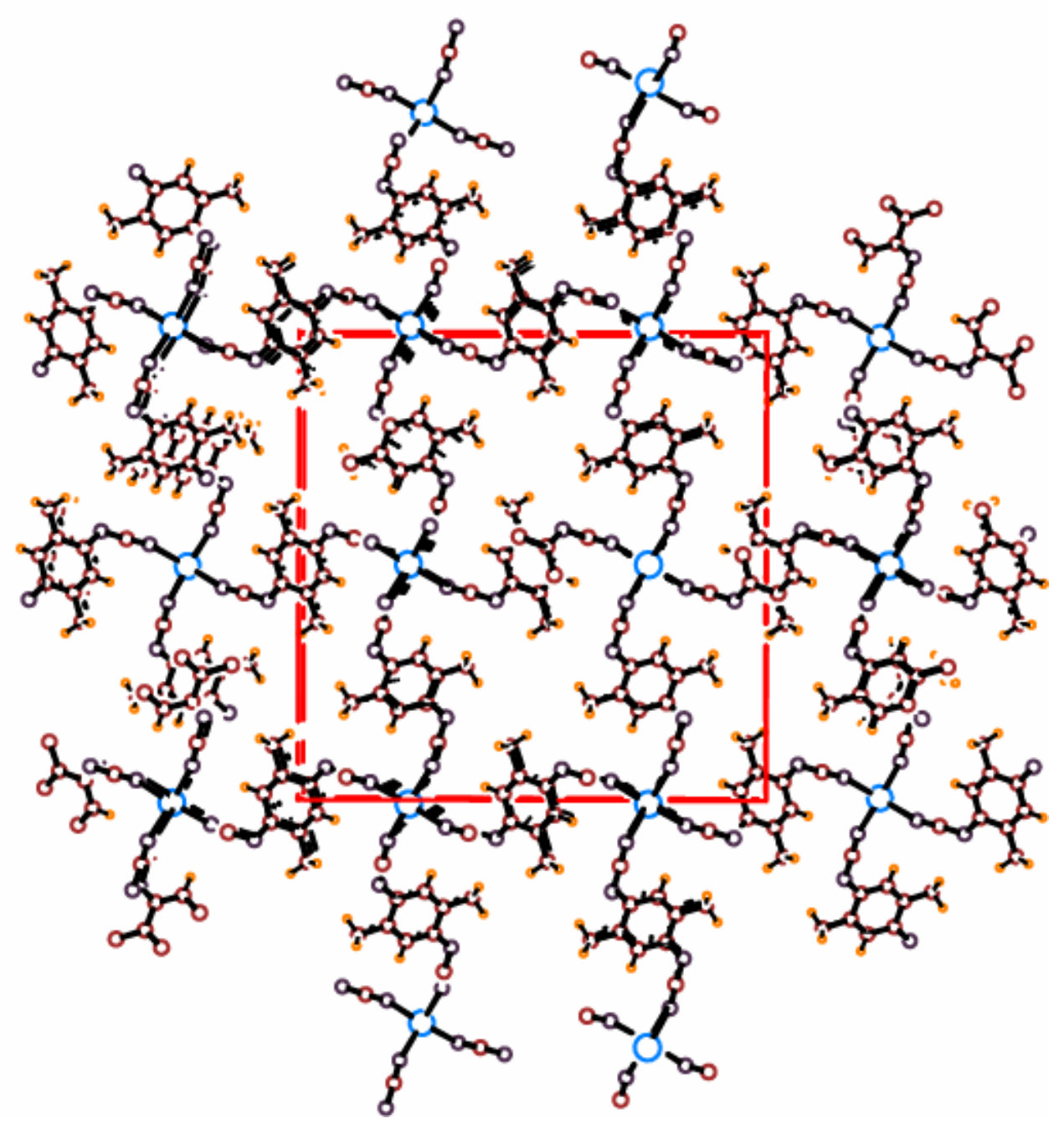

Figure 1. (a) Molecular structure of DM. (b) Crystal structure of $\operatorname{Ag}(\mathrm{DM})_{2}$ viewing down the stacking axis, i.e., the $c$-axis. C: brown; H: orange; N: dark gray; Ag: blue. 
(a)

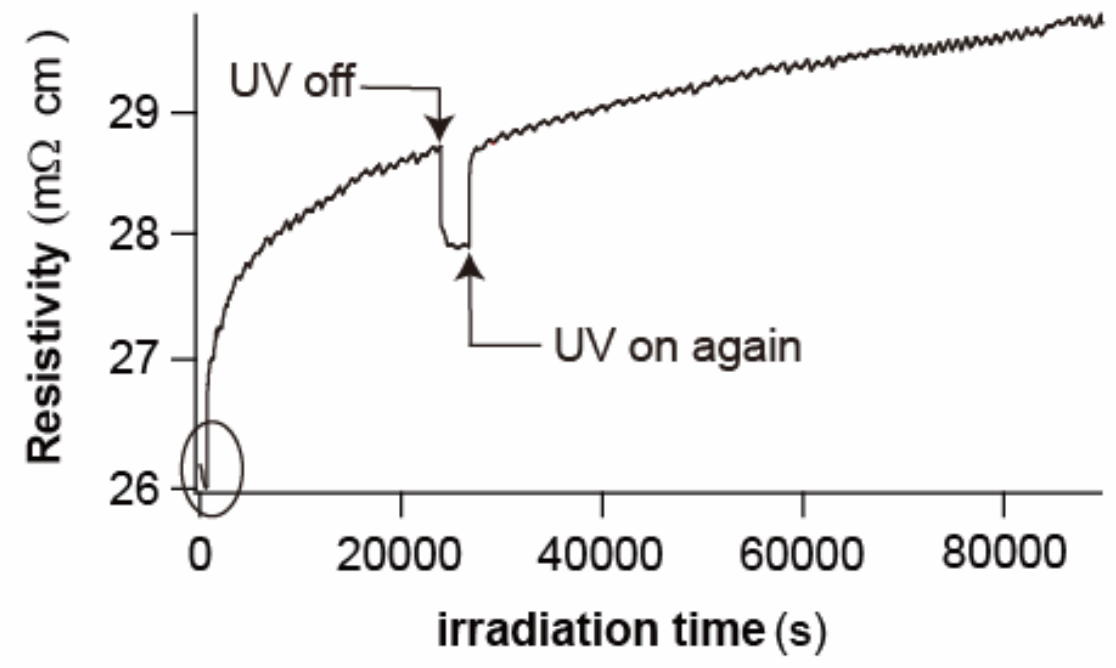

(b)

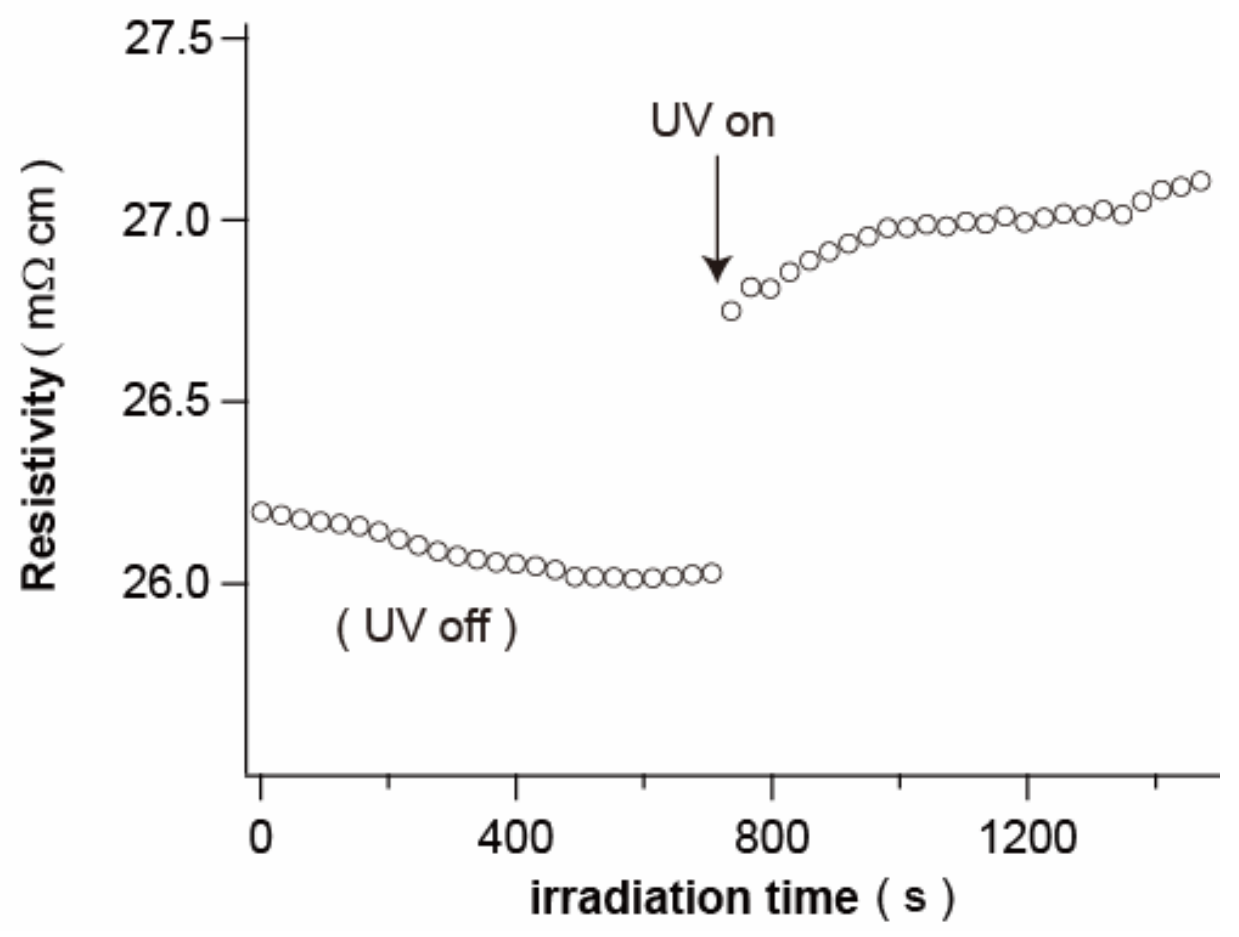

Figure 2. Change in the resistivity of $\mathrm{Ag}(\mathrm{DM})_{2}$ during UV irradiation. (a) Overview, and (b) Close view of the initial stage [encircled part of (a)]. 
that due to the heat involved with the irradiation and that due to the UV light itself. Note that the increase in resistivity due to the heat were of similar degrees between that at irradiation time $t=0$ $\mathrm{s}$ and that at $t=24000 \mathrm{~s}$ (ca. $7 \mathrm{~h}$ from the beginning). This means that the sample reached thermal equilibrium quickly. This resistivity increase indicates that the sample was around $45^{\circ} \mathrm{C}$, which is estimated from the measured temperature (T) dependence of the resistivity ( $\rho$ ) of $\alpha$ around RT.

$$
\frac{d \rho}{d T}=2.5 \times 10^{-5}\left[\Omega \mathrm{cmK}^{-1}\right]
$$

This estimated sample temperature agrees with the actual measured temperature during the irradiation. The results of these electrical measurements are consistent with our previous report [14], in which we measured the temperature dependence of electrical resistivity of a partially irradiated single crystal of $\alpha$. Based on these results and other measurements, it was suggested that $\beta$ should exhibit semiconducting behavior, i.e., a resistivity increase upon decreasing temperature. Considering both of these studies (figure 2), $\beta$ has a slight higher resistivity compared with $\alpha$.

Hereafter we will call the group of UV irradiated samples with irreversibly modified electrical properties $\beta$. At a very initial stage of irradiation, an irradiated sample could contain $\beta$ as well as unreacted $\alpha$. In this case let the name " $\beta$ " indicate exclusively the photochemically modified parts in the solid. As previously reported [23, 24, 27], elemental analysis indicated that all the $\beta$ samples share the same chemical formula as $\alpha$. No irradiation damage or traces of deterioration were observed. Thus, although the possibility of sample deterioration during irradiation cannot be excluded, it is negligible. To determine what causes the irreversible resistivity increase after irradiation, we examined the crystal structure and the chemical and electronic states of $\beta$. In our previous study, a preliminary powder XRD pattern of $\beta$ did not show any peak shift or appearance/extinction of (new) peaks [23, 24, 27]. This indicated that the crystal structure remained static after irradiation. A more detailed examination of the XRD patterns has been carried out on $\beta$ with different irradiation times and the results are shown in figure 3 . The XRD pattern of $\alpha$ was consistent with its reported tetragonal crystal structure [26]. Within $40 \mathrm{~h}$ of irradiation, $\beta$ exhibited diffraction peaks exactly at the same $2 \theta$-angles with those of $\alpha$. Closer examination of these powder patterns revealed that many of the peaks appearing at higher $2 \theta$-angles progressively increased or decreased in relative intensity during irradiation. This indicates that a structural transition is occurring and that the resultant structure is developing with increased irradiation time. It is difficult to distinguish from the respective XRD patterns whether this initial stage of $\beta$ contains residual $\alpha$. Yet it is conceivable that $\alpha$ gradually turns into $\beta$ by way of a mixture state. Therefore, for clarity, let us assume the following two hypotheses: 1 ) this initial 
state ( $\leq 40 \mathrm{~h}$ ) of $\beta$ consists of the starting material $\alpha$ and a new phase $\beta, 2$ ) the mixture ratio of $\alpha$ to $\beta$ is dependent upon irradiation time. This initial stage of $\beta$ is called the $\beta 1$ phase. The $\beta 1$ phase includes all the crystalline solids exhibiting reflection peaks at identical angles $(2 \theta)$ with those of $\alpha$, but with different relative intensities. Such a continuous and subtle structural change suggests that the original lattice of $\alpha$ favors retention of the existing structure while accommodating $\beta 1$ by allowing lattice defects/local deformation. Lattice defects were observed in the magnetic susceptibility of $\beta 1$. When $\alpha$ was exposed to UV-Vis light, local spins increased in number [24]. Because $\beta 1$ is still (semi)conductive with delocalized unpaired electrons on the DM radical anion species, a substantial amount of local spins are considered to originate from these lattice defects. It should be noted here that diffraction peaks due to bulk silver (or Ag nanoparticles) ( $2 \theta=39.4$, 42.2, and higher than 60 deg [21]) were observed in none of the $\beta$ samples. At present, we do not have a definite idea what the lattice defects actually are. However, cleavage (breaking) of some of the Ag-N coordination bonds is highly expected to occur on UV-Vis irradiation, where the defects would be primarily located at this site. Unless all of the four coordination bonds break at an $\mathrm{Ag}^{+}$ ion together with net electron transfer from DM radical anion(s) to the Ag cation, a neutral silver atom would not be liberated, which would be by far less stable than a coordinated silver ion. This assumption is consistent with a proposed production mechanism of $\gamma$ [21]. When a sufficient local density of "unsaturated" (less-than-four-coordinated) silver species is realized, all the remaining coordination bonds would suddenly break to liberate neutral silver atoms and they might assemble to yield elemental silver domains as nanoparticles. There is a room in the crystal lattice for enabling such a series of solid state reactions along the silver ion channels themselves and also along the channels surrounded by methyl groups of DM molecules (figure 1b). Since some $\mathrm{Ag}(0)$ metal-complexes with n-coordination $(n=1-3)$ are studied as oligomers or using matrix isolation [28], the above-mentioned "unsaturated" silver species $\operatorname{Ag}^{\mathrm{x}+}(0 \leq \mathrm{x} \leq 1)$ in an oligomeric chain $(-\mathrm{Ag}-\mathrm{DM}-)_{\mathrm{n}}$ is possible to occur as a metastable state in a crystalline matrix. Further details are under investigation.

Due to the high symmetry of the original unit cell $(\alpha)$, many of the diffraction peaks are overlapped and are related with plural reflection indices. In the $\beta 1$ phase, during irradiation, such overlapped peaks exchanged a part of their intensities with each other, and some intensified while others weakened. Around $60 \mathrm{~h}$ of irradiation, a new series of diffraction peaks suddenly appeared where neither $\alpha$ nor $\beta 1$ showed diffraction peaks. At the same time, there were still traces of diffraction peaks belonging to $\alpha$ or $\beta 1$. Although a discussion on whether $\alpha$ remains in the $\beta$ phase is not possible based on the present data, this result evidently means that a photo-induced structural transition occurred in (a part of) $\alpha$ or $\beta 1$. Chemical reactions in the sample can be excluded, for the chemical composition and IR spectra (discussed below) of $\beta$ were unchanged irrespective of irradiation time. Again, we shall assume hypotheses similar to those of $\beta 1: 1$ ) this 
second stage ( $\geq 40 \mathrm{~h}$ ) of $\beta$ contains another new phase in addition to at least one of the following components: the starting material $\alpha$ and/or $\beta 1,2)$ the mixture ratio of 
(a)

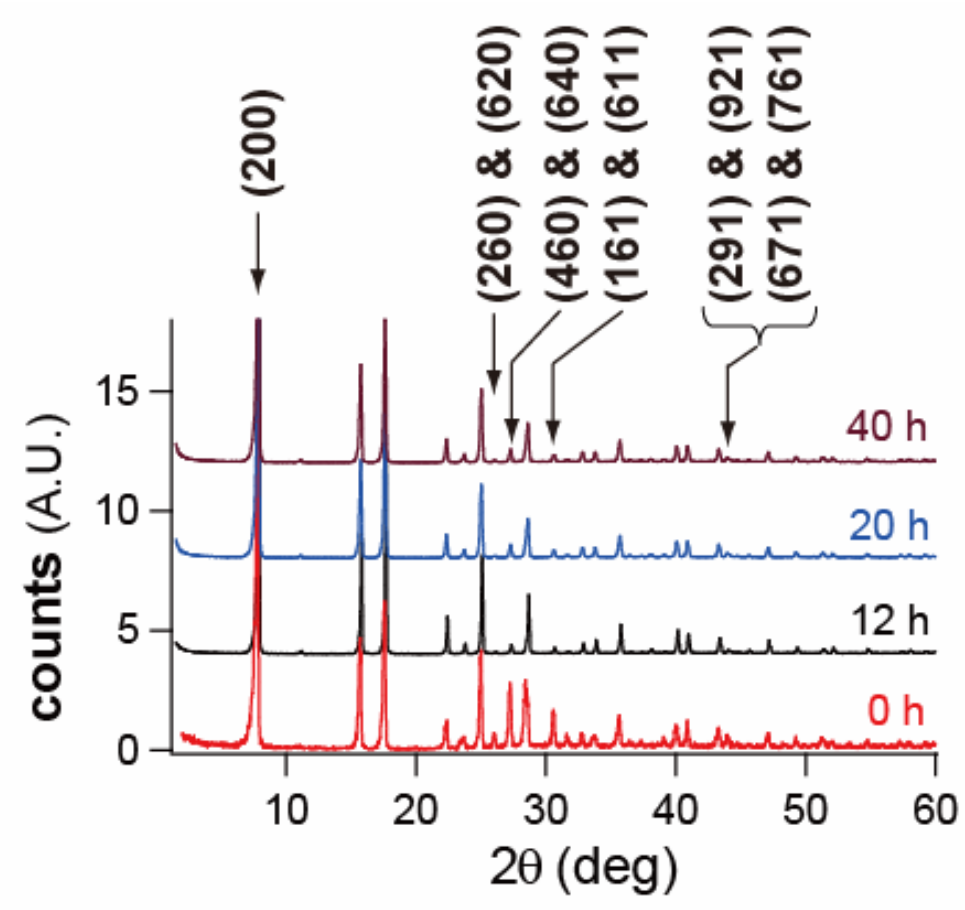

(b)

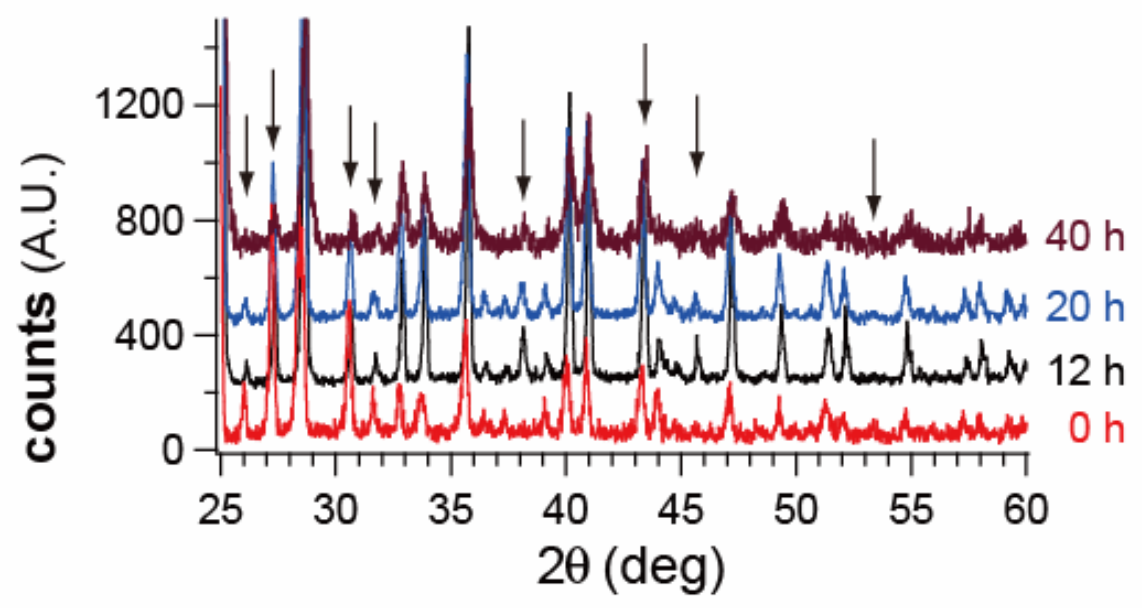

(c)

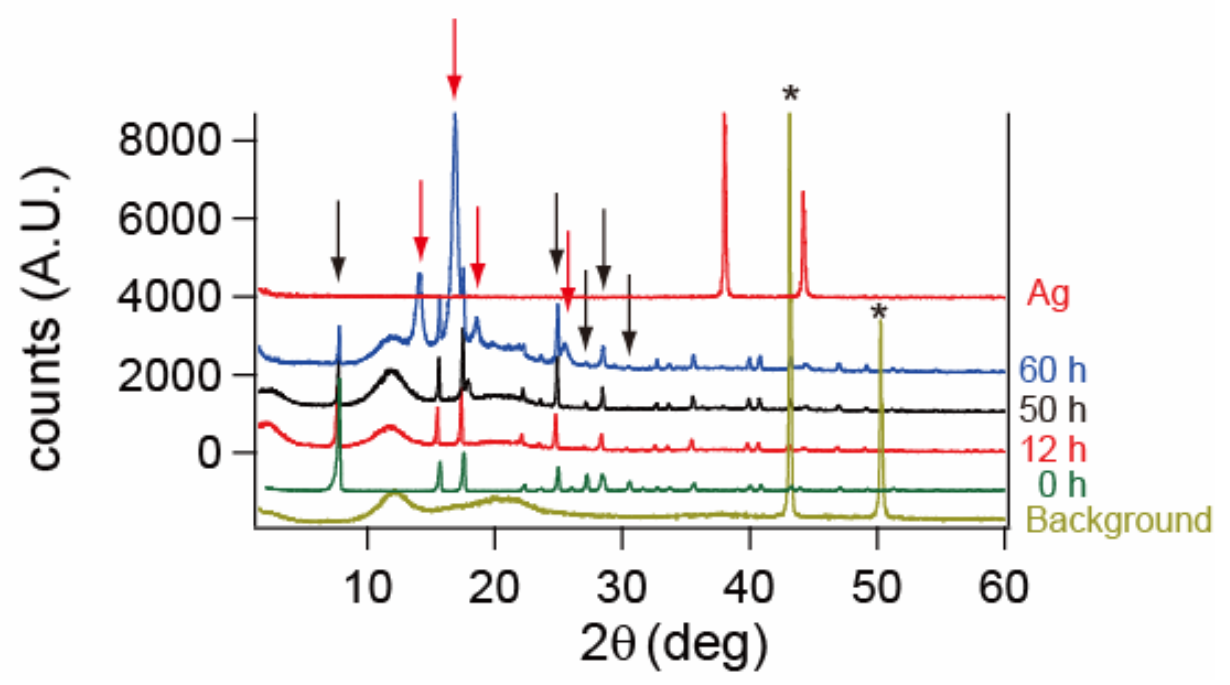


Figure 3. (a) Change in the powder XRD patterns of $\mathrm{Ag}(\mathrm{DM})_{2}$ during UV irradiation from 0-40 h. (b) Close-up view of high 20-angles in (a). (c) (from top to bottom) Ag plate (99.95\%), 60, 50, 12 and $0 \mathrm{~h}$ with background [Scotch tape (broad peaks at 10-20 deg) and base Cu plates (*)]. Black arrows in (b) indicate the diffraction peaks which changed intensity during irradiation. Black and red arrows in (c) indicate the diffraction peaks which progressively weakened (black) or emerged (red) during the irradiation.

this stage is dependent upon irradiation time. The solid $\beta$ of this stage is called the $\beta 2$ phase. The $\beta 2$ phase includes all the crystalline solids exhibiting XRD peaks at different $2 \theta$ from those of $\alpha$ or $\beta 1$. The XRD pattern of the $60 \mathrm{~h}$ sample clearly shows that $\beta 2$ begins with a mixture of $\beta 1$ (or $\alpha$ ) and $\beta 2$. For clarity, let us define $\beta 2$ as the newly emerging crystalline phase with a different structure. The existence of $\beta 1$ and $\beta 2$ phases can be interpreted by the following mechanism. At the initial stage of $\beta$, i.e, in $\beta 1$, the photo-induced modification in the crystal and electronic structures of $\alpha$ will destabilize the lattice. Yet the lattice is robust as stated above with reference to figure 1 , therefore it can sustain the existing structure even with allowing disorder or local deformation. Thus the original lattice of $\alpha$ could accommodate $\beta 1$ by introducing some lattice defects. However, at a certain threshold level of the modification, the instability resulting from defects and local deformation caused by $\beta 1$ would exceed the stabilization energy of the original lattice of $\alpha$. This situation causes a structural transition to $\beta 2$. Thus " $\beta$ ” is divided into two different crystalline phases of $\beta 1$ and $\beta 2$. The actual crystal structures of $\beta 1$ and $\beta 2$ remain to be determined. One could draw a parallel between the results above and other molecular crystalline conducting systems for defects generation under illumination that maintain crystal structure integrity by both of experimental [29] and theoretical [30] approaches. The XAFS of the Ag species has also been measured and will be reported in due course.

In order to clarify the chemical and electronic states of DM in $\beta$, Raman, MALDI, and IR spectra were measured. The MALDI (figure 4) and IR [24] spectra of $\beta 1$ were almost identical to that of $\alpha$. The IR spectra of $\beta 2$ were examined (not shown) and again no clear differences from $\alpha$ or $\beta 1$ were observed. Both spectra indicate that the molecular structure of DM is retained in both $\beta 1$ and $\beta 2$. In the Raman spectra, the $C=C\left(\sim 1620 \mathrm{~cm}^{-1}\right)$ and $C=N\left(\sim 1460 \mathrm{~cm}^{-1}\right)$ stretching modes sensitively dictate the formal charge of DM molecules, even if the charge takes a fractional value [31]. Figure 5 shows a clear difference in the Raman shift of both modes between $\alpha$ and $\beta 1$, which is consistent with an interpretation that the DM species is oxidized by UV irradiation [31]. These Raman shifts correspond to the negative charge of $\sim-0.48$ on each DM radical anion species, deviating from the initial value of -0.50 for the pristine sample. The single peak observed for each stretching mode implies that all the DM molecules are equivalent in $\beta 1$. This is consistent 
with the fact that $\beta 1$ exhibits (semi)conducting properties, which requires delocalization of unpaired electrons of DM radical anions in the crystal.

(a)

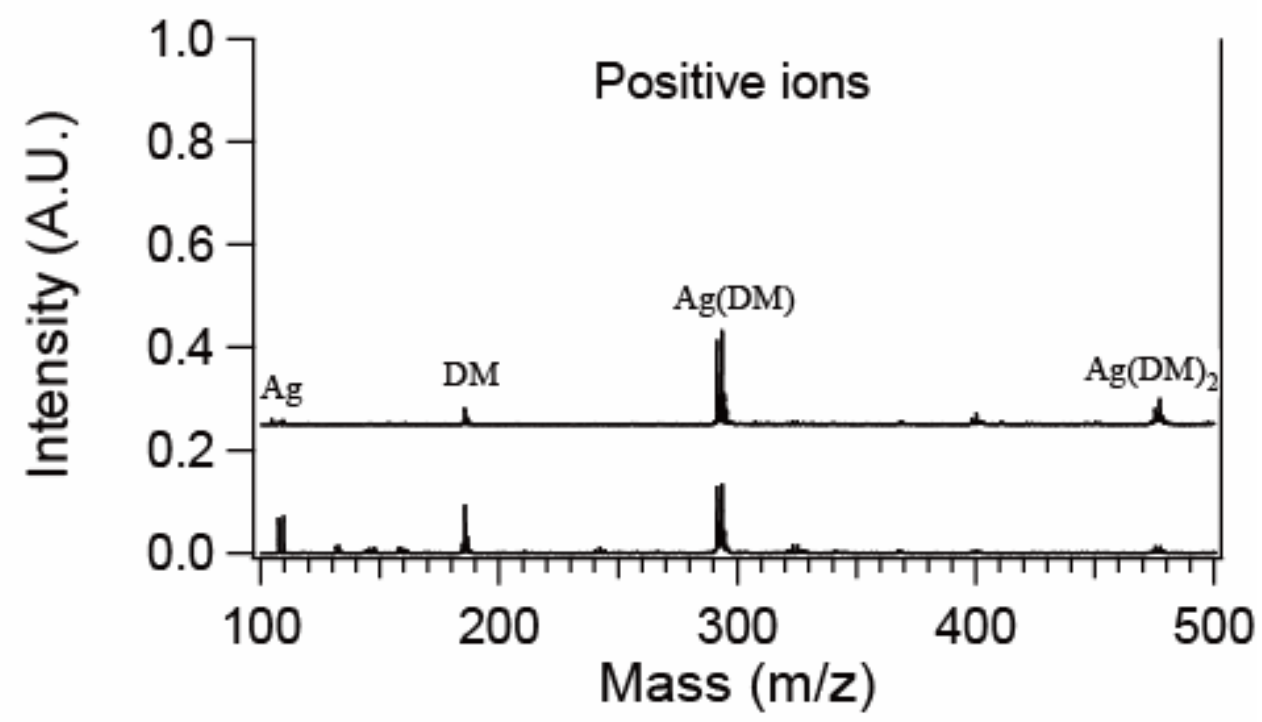

(b)

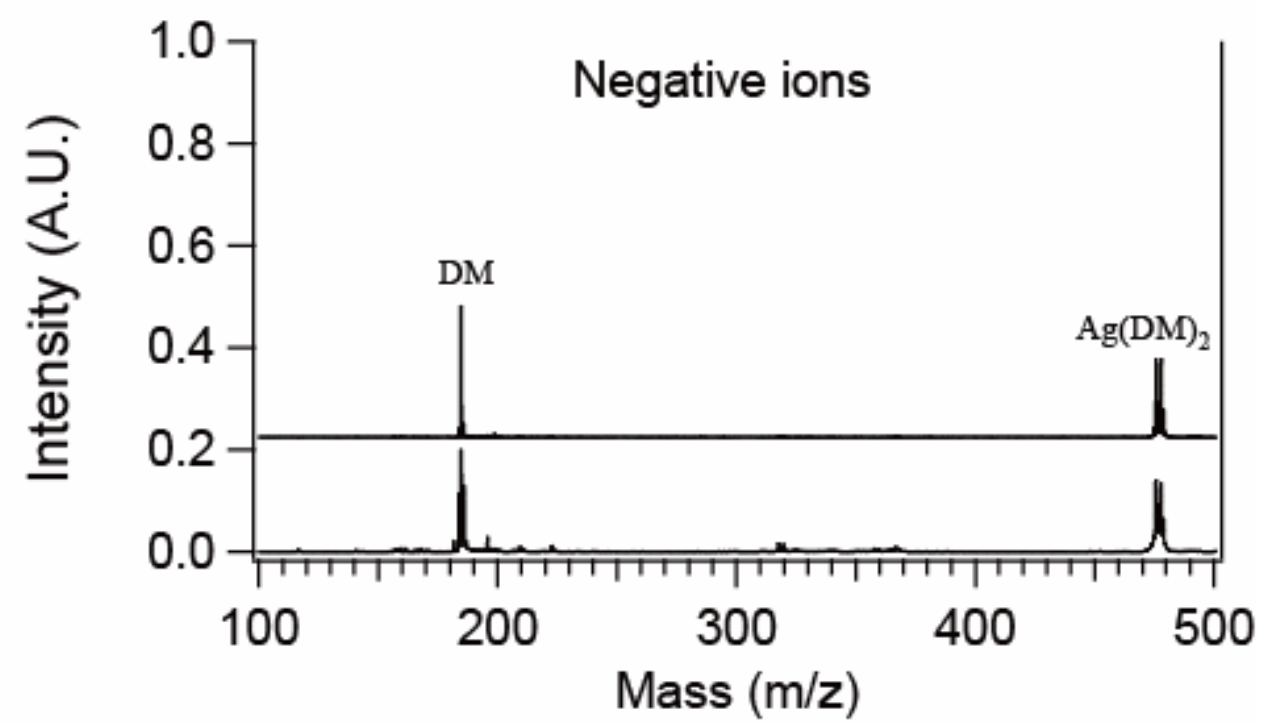

Figure 4. MALDI mass spectra of $\alpha$ (lower) and $\beta$ (upper, irradiated for $12 \mathrm{~h}$ ) of $\operatorname{Ag}(\mathrm{DM})_{2}$; (a) Positive ions, (b) Negative ions. 


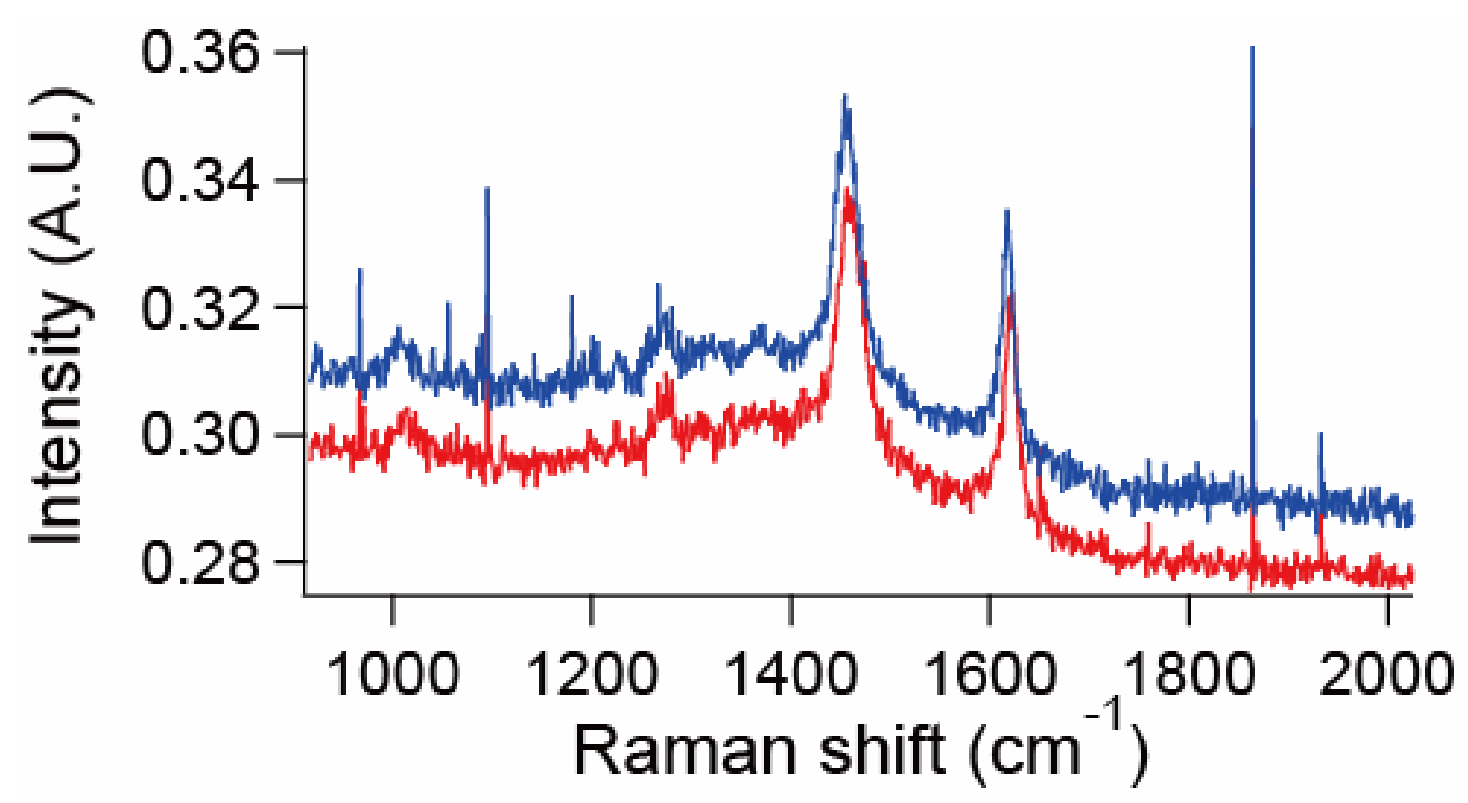

Figure 5. Raman spectra of $\alpha$ (red) and $\beta$ (blue; UV irradiated for 15 h).

Thus far the following points have been discussed: a) UV-Vis irradiation can irreversibly increase the electrical resistivity of $\operatorname{Ag}(\mathrm{DM})_{2}$, b) combining this fact with the temperature dependence of the resistivity measured in our previous work [14], the electrical structure is considered to be modified from that of a metal $(\alpha)$ to that of a semiconductor or a disordered metal $(\beta)$, c) the resistivity increase is not mainly due to deterioration or decomposition of the sample, d) the modified specimen exhibits two kinds of crystalline phases, $\beta 1$ and $\beta 2$, e) as a mixture, the modified sample exhibits a change in structural and electrical properties in a continuous way, f) Raman spectra indicate photo-induced oxidation of the DM radical anion species, but IR and MALDI spectra show only the retention of the molecular structure of the DM radical anion species. Consequently, the relationship between the photo-induced modification of electrical properties and electronic states of the DM molecule responsible for the electrical conduction must be determined. Such an irreversible change in electrical resistivity should be associated with some change in the electronic state of the DM species. Accordingly, information at the molecular level was directly examined by high resolution solid state ${ }^{13} \mathrm{C}$-NMR spectroscopy on $\beta$ samples with different irradiation times (figure 6 ). The spectrum of $\alpha$ is consistent with those of related compounds [32]. All the $\beta$ samples exhibited similar spectra irrespective of irradiation time. These NMR spectra also indicate that the DM species retain the original molecular structure in $\beta$. Going from $\alpha$ to $\beta$, the most obvious change is a dramatic peak shift of carbon \#4. 
(a)

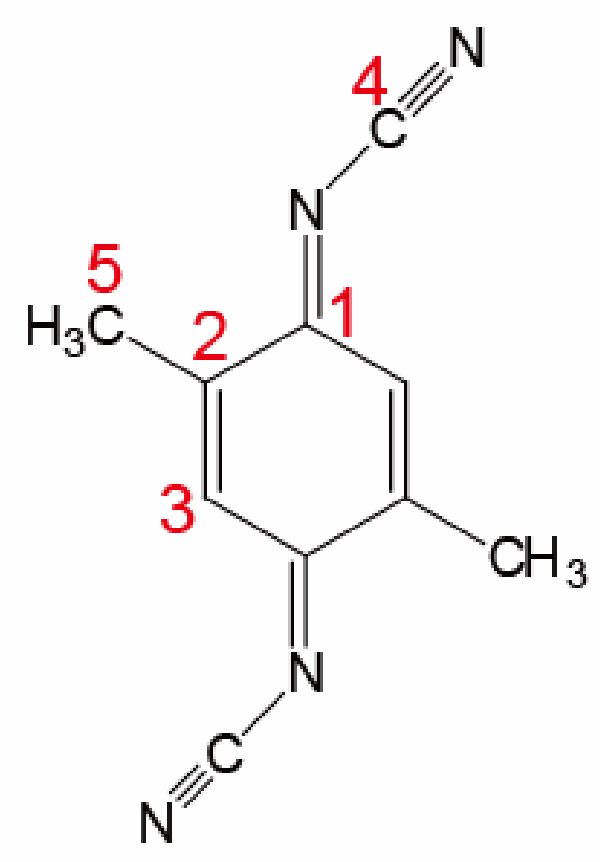

(b)

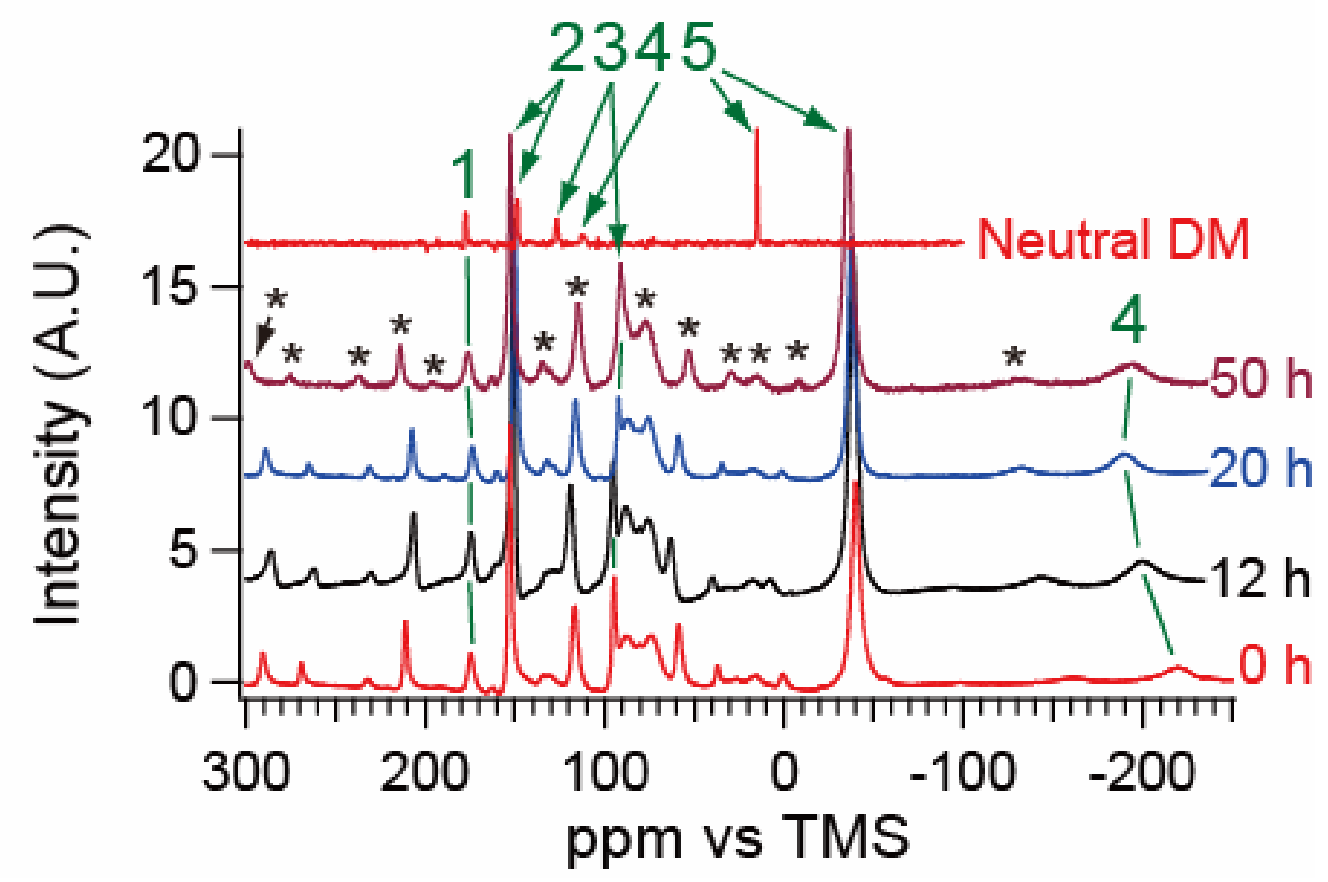

Figure 6. Solid state ${ }^{13} \mathrm{C}-\mathrm{NMR}$ spectra (CPMAS) of $\alpha$ and $\beta$. Spinning side bands are indicated by asterisks.

Among the five independent carbon atoms in DM, this atom (\#4) is less effectively shielded by its valence electrons because of the strong electron withdrawing effects of the two neighboring nitrogen atoms. Thus the resonance peak appears at higher magnetic field. For the same reason, it 
exhibited the largest Knight shift. A Knight shift is a peak shift of the resonance frequency from that of an isolated nucleus, and originates from the shielding effect of conduction electrons. Thus it is a measure of conductivity (resistivity) of the sample, as well as a measure of interaction strength between a particular atom and conduction electrons. Usually the chemical shift of the corresponding atom in a neutral molecule is taken as the origin of the Knight shift, which is 113 ppm vs TMS (tetramethylsilane) in this case (carbon \#4 of DM). Clearly the Knight shift decreases with increasing irradiation. This is consistent with the fact that electrical resistivity of $\beta$ increases with irradiation. Closer examination revealed the remaining peaks also slightly and progressively shifted with irradiation. Saturation was observed in the irradiation effects around 50 h. Similar saturation was also observed after $\sim 50 \mathrm{~h}$ of irradiation in Raman spectra [14]. To summarize the NMR results, it is found that electronic states of the DM species certainly correlate with the change in electrical properties induced by UV irradiation. At present the solid state NMR results are available for $\beta 1$ only, but corresponding ${ }^{13} \mathrm{C}$-NMR measurements on the $\beta 2$ phase are now in progress.

Finally, let us confirm whether heat plays an essential role in the production of $\beta$. Generally speaking, irradiation of light more or less involves some heating effects on samples, and it is difficult to completely exclude the effects in actual experiments. However, it is not difficult to heat a sample completely under dark conditions and examine how heat alone should affect the sample. Accordingly, DSC measurements are informative on this issue. The DSC of $\alpha$ up to $\sim 170{ }^{\circ} \mathrm{C}$ has been recently reported [21]. In DSC charts at various heating rates, there was a single sharp exothermic peak at $155^{\circ} \mathrm{C}$, and no heat flow was detected other than this. This means that nothing occurs in the sample between RT and $155^{\circ} \mathrm{C}$. Thus it was concluded that heat could transform $\alpha$ into an amorphous insulating state named $\gamma$ at $155^{\circ} \mathrm{C}$, but not the semiconducting $\beta$ phase. In other words, the transformation from $\alpha$ to $\beta$ phases is essentially a photo-induced process. This is consistent with the observation that transformation from $\alpha$ to $\beta$ occurred even at $\sim 100 \mathrm{~K}$ in liquid $\mathrm{N}_{2}$ with a similar rate to that at RT. Accordingly, heat does not play an essential role in the production of $\beta$. We have recently measured DSC on $\beta 1$ samples with different irradiation times (12 h, $20 \mathrm{~h}$ and $40 \mathrm{~h}$ ). The results are almost identical with that of $\alpha$ [21]. Such agreement in thermal behavior means that $\alpha$ and $\beta 1$ should be located at an almost identical thermodynamic state. This is consistent with the XRD results in that their crystal structures are almost identical with each other except that more lattice defects are included in $\beta$.

Therefore, it is possible to bring about an irreversible change (increase) in resistivity of $\alpha$ by way of photochemical processes, apart from heating or decomposition of the material. Continuous and fine control of resistivity of $\alpha$ is possible as shown in figure 2. This is because photochemical transformations of solids generally progress from the surface to the interior, which inevitably leaves the solid an inhomogeneous mixture until the irradiation effect saturates the 
entire solid.

\section{Conclusion}

By maintaining the sample temperature below $155^{\circ} \mathrm{C}$ during UV-Vis irradiation, the resistivity of $\beta$ could be varied simply and with fine control. On the other hand, an extremely stable amorphous state $\gamma-\operatorname{Ag}(D M)_{2}$ has also recently been found to be derived from $\alpha$ [21]. The $\beta$ and $\gamma$ phases retain clear-cut outlines of the original crystalline solid and chemical composition. $\beta$ may be utilized as a semiconducting material, while $\gamma$ may contribute to insulating and passivation techniques in the fabrication process of organic devices. Thus the combination of $\alpha, \beta$ and $\gamma$ phases is promising for device applications for the production of regions of any conducting property among metal $(\alpha)$, semiconductor $(\beta)$ or insulator $(\gamma)$ in a single molecular crystal without chemical doping or junction formation with other materials. This creates the possibility of fabrication of FET and IC from a single material of $\mathrm{Ag}(\mathrm{DM})_{2}$.

\section{Acknowledgments}

Elemental analyses were carried out at the Center for Instrumental Analysis, Hokkaido University. T. N. thanks Professors Kenji Mizoguchi (Tokyo Metropolitan University) and Sadamu Takeda (Hokkaido University) for valuable discussions concerning the results of the solid state ${ }^{13} \mathrm{C}$-NMR spectra. T. N. also thanks the research group of Dr. Shigeki Jin at the Open Facilities at Hokkaido University for help with MALDI mass measurements. We thank Dr. Hiroyuki Mayama (Hokkaido University) for helping with the XRD measurements. We thank Profs. Makoto Wakeshima and Yukio Hinatsu (Hokkaido University) for their help with the SQUID measurements. This work is partially supported by the Suhara Memorial Foundation, the Japan Securities Scholarship Foundation, the Saneyoshi Scholarship Foundation, the Hokkaido University Grant Program for Leading Edge Research, a Grant-in-Aid for Scientific Research on Priority Areas of Molecular Conductors (15073207 and 15073101), and a Grant-in-Aid for Scientific Research (A) (15205018).

\section{References}

[1] ed P Batail (2004) Chem. Rev. 104, Thematic Issue for Molecular Conductors. 
[2] O’Neill M and Kelly S M 2003 Adv. Mater. 151135

[3] Otsubo T, Aso Y and Takimiya K 2002 J. Mater. Chem. 122565

[4] Someya T, Sekitani T, Iba S, Kato Y, Kawaguchi H and Sakurai T 2004 Proc. Natl. Acad. Sci. USA 1019966

Someya T, Kato Y, Iba S, Noguchi Y, Sekitani T, Kawaguchi H and Sakurai T 2005 IEEE Transactions on Electron Devices 522502

[5] Potember R S, Poehler T O and Cowan D O 1979 Appl. Phys. Lett. 34405

[6] Hu P, Xiao K, Liu Y, Yu G, Wang X, Fu L, Cui G and Zhu D 2004 Appl. Phys. Lett. 844932

[7] Metzger R M et al 1997 J. Am. Chem. Soc. 11910455

[8] Collier C P, Mattersteig G, Wong E W, Luo Y, Beverly K, Sampaio J F, Raymo M, Stoddart J F and Heath J R 2000 Science 2891172

[9] Wong E W, Collier C P, Bhloradský M, Raymo F M, Stoddart J F and Heath J R 2000 J. Am. Chem. Soc. 1225831

[10] Heath J R and Ratner M A 2003 Physics Today 5643

[11] Kagan C R and Ratner M A 2004 MRS Bulletin 29376

[12] Tao N J 2006 Nature Nanotechnology 1173

[13] Skotheim T A, Elsenbaumer R L and Reynolds J R, ed 1998 Handbook of Conducting Polymers (Dekker, New York), 2nd Ed., rev. and expanded.

[14] Naito T, Inabe T, Niimi H and Asakura K 2004 Adv. Mater. 161786

[15] Farges J -P ed 1994 Organic Conductors (Dekker, New York)

[16] Nalwa H S ed 1997 Handbook of Organic Conductive Molecules and Polymers (Wiley, Stuttgart, Germany), Vol. 1

[17] Williams J M, Ferraro J R, Thorn R J, Carlson K D, Geiser U, Wang H H, Kini A M and Whangbo M -H 1992 Organic Superconductors (Prentice-Hall, Englewood Cliffs, NJ)

[18] Ishiguro T, Yamaji K and Saito G 1998 Organic superconductors (Springer, New York), 2nd Ed

[19] Sleight A W 1988 Science 2421519

[20] D G Ray et al 2005 IEEE Journal of Selected Topics in Quantum Electronics 11539

[21] Naito T, Sugawara H, Inabe T, Kitajima Y, Miyamoto T, Niimi H and Asakura K Uv-vis induced vitrification of a molecular crystal Adv. Func. Mater. in press

[22] Aumüller A and Hünig S 1986 Liebigs Ann. Chem. 1142

[23] Naito T, Sugawara H, Inabe T, Miyamoto T, Niimi H and Asakura K 2006 Mol. Cryst. Liq. Cryst. 455311

[24] Naito T, Sugawara H, Inabe T, Miyamoto T, Niimi H and Asakura K 2007 Photochemical Transformation of Molecular Crystals into Devices Multifunctional Conducting Molecular Materials, ed Saito G, Wudl F, Haddon R C, Tanigaki K, Enoki T, Katz H E 
and Maesato M (RSC Publishing, The Royal Society of Chemistry, Cambridge, UK) pp.181-184

[25] Naito T et al 2001 J. Mater. Chem. 112221

[26] Aumüller A, Erk P, Klebe G, Hünig S, von Schütz J U and Werner H -P 1986 Angew. Chem. Int. Ed. Engl. 25740

[27] Naito T, Sugawara H, Inabe T, Kitajima Y, Miyamoto T, Niimi H and Asakura K $2006 \mathrm{~J}$. Low Temp. Phys. 142383

[28] McIntosh D and Ozin G A 1976 J. Am. Chem. Soc. 983167

Kasai P H and Jones P M 1985 J. Phys Chem. 891147

Chenier J H B, Hampson C A, Howard J A and Mile B 1986 J. Chem. Soc., Chem. Commun. 9730

Chenier J H B, Hampson C A, Howard J A and Mile B 1988 J. Phys Chem. 922745

Chenier J H B, Howard J A, Joly H A, Mile B and Timms P L 1990 J. Chem. Soc., Chem. Commun. 7581

Howard J A, Jones R, Tse J S, Tomietto M, Timms P L and Seeley A J 1992 J. Phys Chem. 969144

[29] Analytis J G, Ardavan A, Blundell S J, Owen R L, Garman E F, Jeynes C, Powell B J 2006 Phys. Rev. Lett. 96177002

Forro L, Schweitzer D and Keller H, 1987 Solid State Commun. 64771

[30] Powell B J and McKenzie R H 2004 Phys. Rev. B 69024519

[31] Lunardi G and Pecile C 1991 J. Chem. Phys. 956911

Meneghetti M, Lunardi G, Bozio R and Pecile C 1991 Synth. Met. 41-43 1775

Yamamoto K, Yakushi K, Hiraki K, Takahashi T, Kanoda K, Meneghetti M and Pecile C 2003 Synth. Met. 135-136 563

[32] Köngeter D, Hentsch F, Seidel H, Mehring M, von Schütz J U, Wolf H C, Erk P and Hünig S 1988 Solid State Commun. 65453

Helmle M, Reiner J, Rempel U, Mehring M,von Schütz J U, Erk P, Meixner H and Hünig S 1991 Synth. Met. 42, 1763 